\title{
Efeitos do tratamento fisioterapêutico na pré-eclampsia
}

\author{
Effects of physiotherapeutic treatment in pre-eclampsia
}

\author{
Viviani Fink Fernandes de Souza ${ }^{[a]}$, Ângela Dubiela ${ }^{[\mathrm{b}]}$, Nelson Francisco Serrão Júnior ${ }^{[\mathrm{c}]}$
}

[a] Acadêmica do curso de Fisioterapia da Universidade Estadual do Centro-Oeste (UNICENTRO), Guarapuava, PR - Brasil, e-mail: vivi_fink@hotmail.com

[b] Docente do curso de Fisioterapia da Universidade Estadual do Centro-Oeste (UNICENTRO), Guarapuava, PR - Brasil, e-mail: angeladubiela@hotmail.com

[c] Docente do curso de Fisioterapia da Universidade Estadual do Centro-Oeste (UNICENTRO), Guarapuava, PR, docente do curso de Fisioterapia da Faculdade Sudoeste Paulista (FSP/Avaré), São Paulo, SP - Brasil, e-mail: nelson_fst@hotmail.com

\section{Resumo}

Introdução: As síndromes hipertensivas gestacionais (SHG) caracterizam uma gestação de alto risco, ocorrendo em $10 \%$ a $22 \%$ das gestantes, e estando a ela relacionadas as intercorrências clínicas maternofetais. Objetivos: Este trabalho teve por objetivo geral realizar uma pesquisa bibliográfica sobre o efeito do tratamento fisioterapêutico na pré-eclampsia e, em específico, avaliar os efeitos do tratamento fisioterapêutico na diminuição da pressão arterial em gestantes que apresentam fatores de risco para desenvolverem a pré-eclampsia. Metodologia: Foi realizada uma pesquisa bibliográfica no período de outubro de 2005 a agosto de 2008, tendo como fonte de pesquisas bases de dados eletrônicos, tais como Bireme, Portal da Pesquisa (Capes) e biblioteca particular. Resultados: Foram encontradas 21 referências, entre livros e periódicos nacionais e internacionais indexados com período de publicação entre 1998 e 2008. Conclusão: Foi verificado que a fisioterapia desempenha um papel importante no tratamento das SHG, principalmente naquelas gestantes que apresentam HAS antes da gestação, pois o exercício aeróbico contribui para que os níveis de pressão arterial sejam controlados, e também cabe ao fisioterapeuta orientar quanto a mudanças nos hábitos de vida, a fim de que os fatores de risco sejam diminuídos.

Palavras-chave: Hipertensão induzida pela gravidez. Pré-eclampsia. Fisioterapia.

\section{Abstract}

Introduction: The pregnancy high pressure syndromes (PHS) characterize a pregnancy of high risk, occurring in $10 \%$ to $22 \%$ of the pregnancies, and being related to it the clinical complications to mother and baby. Objectives: 
This work had for general objective to carry through a bibliographical research on the effect of the physical therapy treatment in the daily before eclampsia and, in specific, to evaluate the effect of the physical therapy treatment in the reduction of the arterial pressure in pregnancy that present risk factors to develop the daily before eclampsia. Methodology: The October of 2005 was carried through a bibliographical research in the period of November of 2009, having as source of research electronic Bireme, Portal of Search databases, and particular library. Results: Twenty-one references between books and index national and international periodic with period of publication between 1998 and 2008 had been found. Conclusions: It was verified that the physical therapy plays an important role in the treatment of PHS, mainly in those pregnancies that present high pressure systemic before the pregnancy, therefore the aerobic exercise also contributes so that the levels of arterial pressure are controlled, and it is also the physiotherapist oriented as the changes in habits of life so that the risk factors are diminished.

Keywords: Hypertension pregnancy-induced. Before eclampsia. Physical therapy.

\section{Introdução}

As síndromes hipertensivas gestacionais (SHG) caracterizam uma gestação de alto risco ocorrendo em 10 a 22\% das gestantes, e estando a ela relacionadas as intercorrências clínicas materno-fetais. Podem ser classificadas em hipertensão crônica $(\mathrm{HC})$, pré-eclampsia/eclampsia $(\mathrm{PE})$, pré-eclampsia sobreposta à hipertensão crônica (PSHC) e hipertensão gestacional (HG) (1, 2).

O termo hipertensão gestacional aguda, denominada pré-eclampsia (pressão arterial $\geq 140 \times 90 \mathrm{mmHg}$ em duas aferições com intervalo de no máximo sete dias), refere-se ao aumento da pressão arterial que se manifesta pela primeira vez durante a gestação (principalmente no segundo trimestre, entre a $16^{\mathrm{a}}$ e a $20^{\mathrm{a}}$ semana), persistindo geralmente até a $12^{a}$ semana do puerpério, e pode ou não estar acompanhada de proteinúria. Sua etiologia é desconhecida e o primeiro sinal clínico se dá pelo aumento da pressão arterial. Entre alguns dos fatores de risco para a hipertensão gestacional pode-se destacar a nuliparidade, SHG em gestação anterior, hipertensão crônica, doença vascular crônica, gestação gemelar, afrodescendência, diabetes, obesidade, doença renal, mola hidatiforme, polidrâmnio, hidropsia fetal, idade materna baixa ou avançada, e ocupações que exijam grande esforço físico $(1,3)$.

Tanto a hipertensão gestacional aguda quanto a hipertensão crônica vão aumentar o risco de complicações na conclusão da gestação, entre elas índice de Apgar baixo no primeiro e quinto minutos, infecção neonatal e prematuridade $(2,4)$.

A princípio o tratamento da hipertensão gestacional consiste em diminuir a pressão arterial da mãe enquanto se aumenta o fluxo sanguíneo para a placenta. Isso se dá com o uso de drogas anti-hipertensivas que vão fazer com que a musculatura lisa das arteríolas periféricas relaxem, reduzindo a resistência vascular. Medidas não farmacológicas também estão indicadas no tratamento da hipertensão gestacional; estas visam a dar orientações relacionadas a hábitos de vida, como diminuir a ingestão de sódio e aumentar a de potássio, abolir a ingestão de álcool e realizar atividades físicas regulares, executadas de forma correta, sob prescrição rigorosa e com orientação profissional (5).

Este trabalho teve por objetivo geral realizar uma pesquisa bibliográfica sobre o efeito do tratamento fisioterapêutico na pré-eclampsia e, em específico, avaliar os efeitos do tratamento fisioterapêutico na diminuição da pressão arterial em gestantes que apresentam fatores de risco para desenvolverem a pré-eclampsia.

\section{Metodologia}

Foi realizada uma pesquisa bibliográfica no período de outubro de 2005 a agosto de 2008, tendo como fonte de pesquisas bases de dados eletrônicos, tais como Bireme, Portal da Pesquisa (Capes) e biblioteca particular. Foram encontradas 50 referências em periódicos nacionais e internacionais indexados com 
período de publicação entre 1998 e 2008. Dessas, dez foram excluídas por estarem em um período anterior a 1998 e por não preencherem os critérios de inclusão, ou seja, referências de gestantes que desenvolveram pré-eclampsia. Os critérios de exclusão foram as referências às outras formas de síndromes hipertensivas gestacionais, bem como mulheres que já apresentavam hipertensão arterial sistêmica (HAS) anterior à gestação. Os descritores utilizados para pesquisa nas bases de dados foram: hipertensão gestacional, pré-eclampsia e fisioterapia.

\section{Desenvolvimento}

O óvulo humano, quando fecundado, precisa se adaptar ao meio interno, para que o organismo da gestante, alterado profundamente pelas repercussões provenientes deste fenômeno, possa permitir sua possível vivência e nutrição. Para isso, ocorrem algumas modificações locais e sistêmicas - as locais são imediatas - para permitir o desenvolvimento do óvulo e sua proteção. Já as modificações sistêmicas são gradativas e ocorrem para proporcionar o indispensável às alterações metabólicas para a formação dos tecidos e constituição de reservas para a vida neonatal (6).

As exigências da gestação atingem os limites de capacidade funcional de muitos órgãos maternos, podendo fazer despontar ou agravar quadros patológicos existentes.

As alterações metabólicas que ocorrem nas gestantes são produzidas de três fatores principais: o primeiro é o sistema enzimático e hormonal, que tem efeito direto sobre os órgãos reprodutores, condicionando ao mesmo tempo reações colaterais como a retenção hídrica; outro fator é expresso pelo volume e circulação no útero, grandemente aumentados, causando alterações estáticas na mulher, nos fenômenos circulatórios sistêmicos e na respiração; e, por último, o aumento das exigências de oxigênio e elementos nutritivos, que provoca diversos distúrbios no metabolismo materno (7).

No período gestacional ocorre uma série de mudanças no funcionamento e nas formas do corpo; talvez em nenhuma outra fase essas mudanças sejam tão significativas em um espaço tão curto de tempo. É importante o conhecimento das adaptações fisiológicas do organismo materno, para que se saiba diferenciar e diagnosticar situações normais de patológicas, para que a gestante possa receber o tratamento adequado. Essas modificações estão presentes em praticamente todos os sistemas, mas nesse estudo convém ressaltar as alterações do sistema cardiovascular.

O volume sanguíneo sofre um aumento progressivo em torno de $35 \%$ a $50 \%$, para que possa suprir as necessidades da parede do útero e da placenta, retornando ao normal dentro de seis a oito semanas após o fim do período gestacional $(6,7)$. Em gestações gemelares o aumento do volume sanguíneo pode atingir a marca de $100 \%$ (4).

O aumento das células plasmáticas é superior ao das células vermelhas, graças ao estímulo hormonal para suprir as demandas de oxigênio, o que faz com que haja uma diminuição no nível de hemoglobina. Esse efeito é conhecido como anemia fisiológica da gestação, o que explica o cansaço e mal-estar relatado por muitas gestantes no início deste período (6).

A progesterona age na musculatura lisa da parede dos vasos, produzindo uma hipotonia, o que consequentemente leva a um aumento da temperatura corporal (4).

Também vai haver um aumento na quantidade de água corpórea e de sódio, em virtude do aumento de estrógeno, que por sua vez ativa o sistema renina-angiotensina-aldosterona, que promove retenção de água e de sódio (5).

O tamanho do coração aumenta, o que faz com que o débito cardíaco se eleve em torno de 30\% a 60\%. Esse aumento é maior em decúbito lateral esquerdo, uma vez que nessa posição o útero impõe menor pressão sobre a aorta. Este ponto deve ser avaliado cuidadosamente pelos fisioterapeutas ao planejarem um programa de treinamento, uma vez que determinada quantidade de exercício produzirá maior débito cardíaco em gestantes do que em não gestantes $(4,6,7)$.

Após o oitavo mês, o débito cardíaco tende a diminuir, por causa da compressão do útero sobre a veia cava inferior, diminuindo o retorno venoso (5). 
A frequência cardíaca pode ter um aumento de 10 a 20 batimentos por minuto ao fim da gestação, voltando ao seu nível normal dentro de até seis semanas do pós-parto (7).

A pressão arterial sofre uma queda nos três primeiros meses da gestação, sendo a diminuição da pressão sistólica menos significativa que a da diastólica (6) -segundo a autora, a primeira sofre uma alteração de 5 a $15 \mathrm{mmHg}$, e a segunda, de 3 a $5 \mathrm{mmHg}$. A pressão arterial sistêmica vai atingir o seu pico mais baixo por volta da $21^{\mathrm{a}}$ semana, e começa a aumentar gradativamente cerca de seis semanas após o nascimento do bebê até atingir o seu nível normal. Essa redução da pressão arterial sistêmica ocorre mesmo com o aumento do débito cardíaco e se deve à distensibilidade venosa $(5,6,7)$.

\section{Hipertensão arterial sistêmica}

Hoje, a HAS apresenta uma prevalência significativa no Brasil, atingindo índices de $22 \%$ a $44 \%$ na população com mais de 18 anos, segundo Gomes (5). É definida como tendo valores de pressão arterial sistólica $>160 \mathrm{mmHg}$, e diastólica $>95 \mathrm{mmHg}$, enquanto os valores de uma pressão arterial normal são citados na literatura como $\leq 120 \mathrm{mmHg}$ para a sistólica e $\leq 80 \mathrm{mmHg}$ para a diastólica. A expectativa de vida da população adulta pode ser diminuída em até $10 \%$ em virtude da HAS $(8,9,10)$.

Os sintomas mais citados no estudo de Peres, Magna e Viana (11) são dor de cabeça, dor no corpo e cansaço, e após a confirmação do diagnóstico de HAS grande parte dos indivíduos relataram apresentar dor de cabeça e na nuca, coração acelerado, juntamente com pontada no peito, tontura, dor no corpo e nas veias.

Entre os fatores de risco para HAS incluem-se:

a) idade: graças às alterações da musculatura e do tecido de revestimento dos vasos, que são características do envelhecimento;

b) sexo: fator que atinge mais os homens que as mulheres, embora a mudança de hábitos femininos venha fazendo essa diferença diminuir;

c) raça: os negros são mais acometidos, principalmente na faixa etária dos 35 a 44 anos;

d) obesidade, estresse e sedentarismo: a atividade física realizada pelo menos três vezes por semana por pelo menos 30 minutos melhora a condição física e consequentemente diminui o risco de ser desenvolvida a HAS;

e) consumo de álcool: aumenta a pressão arterial em cerca de $2 \mathrm{mmHg}$ a cada $30 \mathrm{ml}$ de álcool etílico ingerido diariamente;

f) tabagismo, uso de anticoncepcionais e dieta rica em sódio e gorduras.

Em âmbito profissional, a HAS aparece num índice menos significativo na classe social mais alta e naqueles indivíduos que possuem nível de escolaridade mais alto (10).

O tratamento para a HAS se divide em não farmacológico e farmacológico. A primeira opção de tratamento apresenta efetividade comprovada para diminuir os índices de pressão arterial. Entre estas medidas estão a redução do peso corporal, da ingestão de sal e do consumo de bebidas alcoólicas, assim como a prática regular de exercícios físicos. Este último tem sido incorporado nas últimas décadas como um dos principais recursos terapêuticos para o paciente hipertenso, em conjunto com o tratamento medicamentoso e as mudanças de hábitos de vida. Contudo, o exercício físico deve ser realizado de forma correta e tendo efetividade comprovada apenas para as recomendações mais rigorosas; seu objetivo é aumentar a capacidade funcional para que o paciente possa dispor de uma melhora na qualidade de vida e do prognóstico (5).

Do ponto de vista farmacológico, podem ser administrados ao paciente diuréticos, betabloqueadores, simpatolíticos de ação central, antagonistas dos canais de cálcio, inibidores da enzima conversora da angiotensina e antagonistas do receptor da angiotensina II. Quando essas drogas já não são capazes de controlar a pressão, a dose pode ser aumentada ou então adicionada uma segunda droga (12). 


\section{Pré-eclampsia}

É uma das principais causas de morbidade e mortalidade materna, podendo se manifestar de forma pura, sem antecedentes de hipertensão crônica, ou sobreposta a uma doença hipertensiva subjacente, geralmente acometendo primigestas, com maior incidência em casos de gestações múltiplas. As intercorrências clínicas durante o período reprodutivo da mulher não se expressa apenas nos índices de mortalidade fetal, mas também sacrificam muitas vidas maternas (4).

A pré-eclampsia é uma das síndromes hipertensivas gestacionais, podendo ser observada a partir da $20^{2}$ semana de gestação, apresentando como características a pressão arterial em níveis iguais ou maiores que $140 \times$ $90 \mathrm{mmHg}$, edema e proteinúria, esta última podendo aparecer posteriormente, à medida que a gestação evolui. Esta patologia, além da tríade clássica, pode apresentar alguns sintomas que exigem maior atenção do profissional da saúde que está acompanhando a gestante e que podem auxiliar no diagnóstico, como cefaleia, visão turva, dor abdominal, náuseas e vômito. A sua forma mais grave pode ser caracterizada pela presença de vasoespasmo ao exame de fundo de olho, ganho de peso acima de $400 \mathrm{~g}$ por semana e volume urinário inferior a $600 \mathrm{~mL}$ por dia (4).

A pré-eclampsia é responsável por complicações em $2 \%$ a $8 \%$ das gestações. Em se tratando de mortalidade materna, este número é ainda maior, podendo atingir $10 \%$ a $15 \%$ das gestantes, de acordo com Ruano, Fontes e Zughaib (13), apresentando um índice menor em países desenvolvidos (14). Pode também afetar o feto, causando hipóxia, restringir seu crescimento intrauterino, levar a um parto prematuro e, em virtude da associação desses fatores, a um alto índice de mortalidade perinatal (15).

Sua etiologia ainda é desconhecida, embora a partir dos anos 80 tenham começado a ser desenvolvidos estudos que mostram que um metabolismo desordenado do ácido araquidônico poderia estar associado à sua patogenia, assim como uma deficiência de prostaciclina intravascular (vasodilatador) e tromboxano (vasocostritor) produzido em grande escala (13).

Sobre a sua fisiopatolgia, pode-se afirmar que é caracterizada por vasoespasmo generalizado associado à hipovolemia. A paciente com pré-eclampsia vai responder de forma mais intensa à angiotensina II, adrenalina e noradrenalina (8). Este vasoespasmo generalizado e a ativação da coagulação estão relacionados aos danos endoteliais mediados à ativação do sistema imunológico materno.

A incidência dessa complicação abrange primigestas, pacientes com história familiar, antecedentes gestacionais e gestantes com aumento da massa trofoblástica (gestação múltipla, gestação molar, triploidia fetal). O aparecimento de convulsões em pacientes com pré-eclampsia, sem causa identificável, caracteriza um quadro de eclampsia (9).

Seu diagnóstico é realizado por meio de exames clínicos e complementares. Um exame amplamente utilizado nas gestantes de risco é a ultrassonografia com Doppler, que tem sido usada como um teste para se detectar a pré-eclampsia precocemente, por volta do primeiro e segundo trimestre da gestação, antes mesmo de aparecerem os sinais clínicos. Este exame vai verificar a velocidade de fluxo sanguíneo na artéria uterina (16).

Dentre os principais fatores de risco para a pré-eclampsia ainda estão extremos de idade materna, cor, obesidade, diabetes, doença vascular crônica, pré-eclampsia em gestação anterior, gestação gemelar, doença renal, excesso de esforço físico ou psíquico, hidropsia fetal $(1,4)$. Barton e Sibai $(16)$ relatam que pode haver associação entre infecções do trato urinário e maior propensão à pré-eclampsia. Segundo Neojatizadeh, Stobdan, Malhotra e Pasha (17), mulheres que apresentam histórico familiar para esta síndrome têm risco maior de desenvolvê-la.

Recomenda-se também que mulheres que apresentem mais de um fator de risco para a pré-eclampsia façam consultas pré-natal mais frequentes do que aquelas que não são tão suscetíveis a esta síndrome (16).

Quando não tratada, ou quando a gestação não é interrompida, sua tendência é evoluir para as formas mais graves de síndromes hipertensivas gestacionais, como a síndrome de Hellp e a eclampsia (18, 19).

\section{Tratamento da pré-eclampsia}

O objetivo principal do tratamento da pré-eclampsia é reduzir a pressão sanguínea materna, ao passo que o fluxo sanguíneo para a placenta é aumentado. As drogas mais utilizadas nesses casos são a hidralazina e 
a metildopa, pois promovem relaxamento da musculatura lisa das arteríolas periféricas e também diminuem a resistência dos vasos (1).

Duley (14) afirma que óleo de peixe também apresenta um resultado promissor no tratamento da pré-eclampsia.

Já a fisioterapia pode atuar de forma preventiva, diminuindo os fatores de risco antes da concepção, melhorando a saúde materna e utilizando-se do exercício físico como recurso terapêutico, que possui papel fundamental na recuperação de pacientes com disfunções cardiovasculares (16). O objetivo neste caso é diminuir a frequência cardíaca de repouso, e da pressão arterial antes, durante e depois do exercício, mostrando assim a sua eficiência como terapia não farmacológica e que o fisioterapeuta pode atuar positivamente no tratamento da pré-eclampsia (5).

Um exercício de baixa intensidade é capaz de diminuir os níveis pressóricos significativamente, isto porque também vai reduzir o valor do débito cardíaco, o que pode ser explicado pela queda da frequência cardíaca de repouso. É interessante que essas pacientes iniciem programas de exercícios regulares depois de terem passado por uma criteriosa avaliação médica. Esses exercícios, segundo a Sociedade Brasileira de Cardiologia, devem ser realizados entre três e seis vezes por semana, com no mínimo 30 minutos, evoluindo para até 60 minutos de duração. A frequência cardíaca deve estar entre $60 \%$ e $80 \%$ da máxima e o consumo máximo de oxigênio entre $50 \%$ e $70 \%$ (20).

Os mesmos exercícios prescritos e realizados no puerpério também apresentam benefícios para gestantes com pré-eclampsia e estes não alteram a pressão arterial; entre eles estão caminhada, corrida, natação e hidroginástica (4).

\section{Discussão}

É reconhecido que a melhora da aptidão física pode contribuir significativamente para a saúde, elevando o nível da qualidade de vida $(21,22)$. Dentre as atividades físicas que podem melhorar a saúde, a prática de exercícios físicos vem sendo recomendada pelas principais agências normativas da atividade física, como o American College of Sports Medicine (ACSM) (23,24) e a American Heart Association (AHA) (25), por causa da sua relativa segurança, mesmo em populações ditas especiais, como as gestantes, em especial aquelas com pré-eclampsia.

Existe uma escassez de informação concernente às respostas e adaptações cardiovasculares aos exercícios físicos na pré-eclampsia. Conclusões englobando essas respostas cardiovasculares são mais complicadas por vários fatores, sendo o principal deles referente aos efeitos do volume e intensidade que o treinamento tem sobre a resposta fisiológica e adaptações de longo tempo (26). Na literatura, identifica-se um predomínio de estudos que buscam relacionar a segurança cardiovascular ao tipo de contração envolvida no exercício (estática ou dinâmica), principalmente em indivíduos pertencentes a grupos de risco (como gestantes com pré-eclampsia) ou atletas (27).

Ganem e Castiglia (28) relatam que a hipertensão arterial está relacionada ao aumento da resistência vascular resultante da hiperatividade simpática e da hipersensibilidade à adrenalina, à noradrenalina e à angiotensina II. Tais autores ainda afirmaram que a lesão endotelial imunorreativa ocasiona agregação plaquetária e de fibrina no leito vascular, e o aumento da extensão do comprometimento renal demonstra o agravamento da doença, sendo necessário o tratamento farmacológico.

Em relação ao tratamento da pré-eclampsia com drogas anti-hipertensivas, Ferrão, Pereira, Gersgorin, Paula, Corrêa e Castro (1) citam a hidralazina e a metildopa para diminuir a pressão sanguínea e aumentar o fluxo de sangue para a placenta. Essas drogas agem promovendo um relaxamento da musculatura lisa das arteríolas e diminuindo a resistência dos vasos.

Wannmacher (19) em seu estudo relata benefícios do labetalol e nifedipino, além da hidralazina, sendo o primeiro mais utilizado por preservar o fluxo uteroplacentário. Comparando o nifedipino com a hidralazina, o primeiro apresentou menor estresse fetal e controle da pressão arterial mais prolongado, além de ser administrado via oral. Já correlacionando a hidralazina com labetalol, a primeira provocou maiores reações adversas na mãe e menor bradicardia neonatal. 
Duley (14) diz que doses baixas de aspirina, em conjunto com outros agentes antiplaquetários, ajuda a prevenir até $19 \%$ dos casos de pré-eclampsia, por esta síndrome estar associada à deficiência de produção da prostaciclina e excesso de tromboxano. Barton e Sibai (16) também sugerem a administração de baixas doses de aspirina para prevenir a pré-eclampsia.

Em virtude do grande número de fatores de risco para a pré-eclampsia, estes devem ser avaliados e tratados antes da gestação - sendo o principal deles a hipertensão crônica (16) - evitando o grande número de medicações, que podem trazer efeitos colaterais tanto para a gestante como para o bebê.

A intervenção fisioterapêutica no tratamento da pré-eclampsia visa a atingir o que foi proposto por Barton e Sibai (16), que é reduzir os níveis pressóricos antes da gestação, visto que, segundo Baracho (4), o exercício físico é contraindicado para gestantes que apresentam alterações na pressão arterial. Duley (14) cita a prática de exercícios aeróbicos e a redução da ingestão de sal como medidas efetivas para a diminuição da pressão arterial.

Diversos autores encontraram aumento abrupto da FC nas primeiras quatro semanas de gestação, seguido por um aumento gradual, atingindo um platô de aproximadamente 15 batimentos por minuto acima dos valores da FCR no estado não gravídico $(26,27,28)$. Esse aumento pode ser causado por ajustes vasculares e hemodinâmicos decorrentes da gestação (28).

Um estudo de Pivarnik et al. (29) comparou um grupo de gestantes que praticou exercício ao longo da gestação com um grupo de gestantes sedentárias. $\mathrm{Na} 25^{\mathrm{a}}$ e $36^{\mathrm{a}}$ semanas de gestação e na $12^{\mathrm{a}}$ semana pós-parto, os dois grupos foram testados em cicloergômetro, com a FC fixa em $140 \mathrm{bpm}$. Os resultados demonstraram que as gestantes treinadas atingiram cargas mais altas, para uma mesma FC, do que as sedentárias, e ainda apresentaram um volume sistólico de repouso maior durante o exercício. Apesar de o grupo de gestantes treinadas ter apresentado um aumento da FC, esse aumento foi menor do que o das gestantes não treinadas.

A diminuição da PA durante imersão em repouso, em gestantes, é descrita na literatura (29, 30), podendo ser explicada pelo aumento do tônus vagal $(31,32)$ e pela ação da pressão hidrostática que age no edema de mulheres grávidas. O redirecionamento sanguíneo produz grande diurese a partir da estimulação dos receptores que conduzem a ajustes reflexos hormonais e neurais e provocam diurese e natriurese. Esse sinal é imediato aos receptores e conduz, consequentemente, a uma queda na pressão sanguínea sistólica e diastólica (33).

Hartmann et al. (33) compararam as respostas de PA em gestantes em repouso e durante exercício na água e na terra, e verificaram que a imersão não afetou significativamente a pressão arterial na água durante o repouso e o exercício; porém, tanto a PAS quanto a PAD baixaram mais no pós-exercício da água do que da terra.

McMurray et al. (34) testaram 12 mulheres grávidas, com o objetivo de determinar o efeito na gestação das respostas cardiovasculares durante exercício aquático e durante imersão. A amostra permaneceu imersa, em repouso, por 20 minutos e outros 20 minutos pedalando em cicloergômetro a $60 \%$ do VO2 máximo predito. A temperatura da água foi de $30^{\circ} \mathrm{C}$ e as gestantes foram testadas nas semanas 15,25 e 35 de gestação e 8-10 semanas após o parto. Os resultados mostraram que a FC a uma mesma exigência metabólica é mais baixa no exercício na água do que na terra. A combinação de exercício aquático e gestação resulta em maior aumento do débito cardíaco do que o esperado em terra. Este estudo demonstra que a resposta de FC e de PA durante o exercício na água é diferente do na terra; por isto, sugere que não é indicado usar as mesmas zonas de FC de treinamento que as do exercício em terra.

Alguns autores (35) não evidenciam papel fundamental do exercício aeróbico regular, durante a gravidez, na prevenção da hipertensão gestacional. Entretanto, Tomoda et al. (36) avaliaram dois grupos de gestantes, um grupo que caminhava 25 minutos, três vezes por semana, com FC em torno de $120 \mathrm{bpm}$, e outro grupo que não praticou exercício físico. Após testarem os grupos na $12^{\mathrm{a}}, 22^{\mathrm{a}}, 29^{\mathrm{a}}$ e $36^{\mathrm{a}}$ semana gestacional e um mês pós-parto, os autores concluíram que o exercício moderado foi capaz de prevenir a hipertensão gestacional, já que 22,5\% do grupo que não se exercitou desenvolveu hipertensão gestacional, enquanto no grupo que praticou exercício não houve nenhum caso.

Sorensen et al. (37) verificaram em seu estudo que mulheres que se engajaram em qualquer atividade física regular no início da gestação, comparadas com mulheres inativas, tiveram uma redução de 35\% do risco de desenvolver pré-eclampsia, e concluíram que a atividade física regular, particularmente quando "realizada" durante o ano anterior à gravidez e durante o início da gestação, é associada com um risco reduzido de pré-eclampsia. 
Weissgerber et al. (38), estudando o papel da atividade física regular na prevenção da pré-eclâmpsia, sugerem que o exercício regular pré-natal pode prevenir ou opor-se à progressão desta doença.

Os autores citados anteriormente avaliaram os efeitos agudos da imersão na resposta da FC e da PA. Já Tomoda et al. (36), Sorensen et al. (37) e Weissgerber et al. (38) estudaram o efeito crônico do exercício na prevenção da hipertensão gestacional e concluíram que este exerce um papel importante nas adaptações cardiorrespiratórias $(39,40)$.

Monteiro e Filho (20) dizem que o exercício físico de intensidade leve a moderada é capaz de controlar a pressão arterial sem medidas farmacológicas associadas. Em seu estudo afirma também que a Associação Brasileira de Cardiologia recomenda a prática de exercícios físicos de três a seis vezes por semana, por pelo menos 30 minutos. Gomes (5) também atesta que o exercício físico é excelente auxiliar na redução dos níveis de pressão arterial e de frequência cardíaca, o que mostra que a intervenção fisioterapêutica é bastante efetiva no tratamento da HAS.

Uma intervenção mais agressiva e que pode ser evitada com a prevenção e diminuição dos fatores de risco da pré-eclampsia é a interrupção da gestação por volta da $34^{a}$ ou $37^{a}$ semana (19). Esta prática pode trazer efeitos deletérios para o bebê, principalmente se não estiver a termo.

O papel do treinamento físico em gestantes ainda é pouco conhecido, graças aos poucos estudos de qualidade disponíveis. Apesar de este estudo ser um trabalho de pesquisa bibliográfica, pensamos que futuras investigações devem ser realizadas para esclarecer melhor o papel do exercício físico no comportamento hemodinâmico ao longo da gestação.

\section{Considerações finais}

Neste estudo verifica-se que a pré-eclampsia apresenta uma grande incidência e que, além disso, pode trazer tanto à mãe quanto ao bebê graves intercorrências, quando não tratadas de forma adequada, podendo levar um ou outro e até mesmo ambos a óbito. Com o conhecimento dos fatores de risco, pode ser desenvolvido um tratamento preventivo para evitar que esta síndrome se instale, provocando maiores complicações.

Nesse aspecto a fisioterapia desempenha um papel importante, principalmente em gestantes que apresentam HAS antes da gestação, pois o exercício aeróbico, segundo Barton e Sibai (16), contribui para que os níveis de pressão arterial sejam controlados. Também cabe ao fisioterapeuta orientar quanto a mudanças nos hábitos de vida, a fim de que os fatores de risco sejam diminuídos. Seria interessante que houvesse mais estudos salientando a importância do tratamento preventivo da pré-eclampsia, bem como uma orientação adequada às gestantes do grupo de risco a fazer consultas pré-natal com maior frequência.

\section{Referências}

1. Ferrão MHL, Pereira ACL, Gersgorin HCTS, Paula TAA, Corrêa RRM, Castro ECC. Efetividade do tratamento de gestantes hipertensas. AMB Rev Assoc Med Bras. 2006;52:390-4.

2. Oliveira CA, Lins CP, Sá RAM, Netto HC, Bornia RG, Silva NR, et al. Síndromes hipertensivas da gestação e repercussões perinatais. Revista Brasileira de Saúde Materno Infantil 2006. Rev Bras Saúde Matern Infant. 2006;6(1):93-8.

3. Costa HLFF, Costa CFF, Costa LOBF. Idade materna como fator de risco para a hipertensão induzida pela gravidez, análise multivariada. Rev Bras Ginecol Obstet. 2003;25(9):631-5.

4. Finkelstein L, Bgeginski R, Tartaruga MP, Alberton CL, Kruel LFM. Comportamento da freqüência cardíaca e da pressão arterial, ao longo da gestação, com treinamento no meio líquido. Rev Bras Med Esporte. Rev Bras Med Esport. 2006;12(5):376-80.

5. Gomes PA. Intervenção fisioterapêutica no paciente hipertenso [monografia]. Araras: Faculdade de Fisioterapia Heminio Ometto; 2003. 
6. Wolfe LA, Davies GAL. Canadian guidelines for exercise in pregnancy. Clin Obstet Gynecol. 2003;46(2):488-95.

7. Hermida RC, Ayala DE, Mojón A, Fernández JR, Alonso I, Silva I, et al. Blood pressure patterns in normal pregnancy, gestational hypertension, and preeclampsia. Hypertension. 2000;36(2):149-58.

8. Barrileaux PS, Martin JN. Hypertension therapy during pregnancy. Clin Obstet Gynecol. 2002;45(1):22-34.

9. Katz VL. Exercise in the water during pregnancy. Clin Obstet Gynecol. 2003;46(2):432-41.

10. Pessuto J, Carvalho EC. Fatores de risco em indivíduos com hipertensão arterial. Rev latino-am Enfermagem. 1998;6(1):33-9.

11. Péres DS, Magna JM, Viana LA. Portador de hipertensão arterial: atitudes, crenças, percepções, pensamentos e práticas. Rev Saúde Pública. 2003;37(5):635-42.

12. Mion D, Pierin AMG, Guimarães A. Tratamento da hipertensão arterial: respostas de médicos brasileiros a um inquérito. Rev Assoc Méd Brasil. 2001;47(3):249-54.

13. Ruano R, Fontes RS, Zugaib M. Prevention of preeclampsia with low-dose aspirin - a systematic review and metaanalysis of the main randomized controlled trials. Clinics (São Paulo). 2005;60(5):407-14.

14. Duley L. Pre-eclampsia and hypertensive disorders of pregnancy. Br Med Bull. 2003;67:161-76.

15. Coelho TM, Martins MG, Viana E, Mesquita MRS, Camano L, Sass N. Proteinuria nas síndromes hipertensivas da gestação: prognóstico materno e perinatal. Rev Assoc Med Brás. 2004;50(2): 207-13.

16. Barton JR, Sibai BM. Prediction and prevention of recurrent preeclampsia. Obstet Gynecol. 2008;112(2 Pt 1):359-72.

17. Nejatizadeh A, Stobdan T, Malhotra N, Pasha MAQ. The genetic aspects of preeclampsia: achivements and limitations. Biochem.Genet. 2008;46(78):451-79.

18. Peraçoli JC, Parpinelli MA. Síndromes hipertensivas da gestação: identificação de casos graves. Rev Bras Ginecol Obstet. 2005;27(10):627-34.

19. Wannmacher L. Manejo da hipertensão arterial na gestação: o pouco que se sabe. Organização Pan Americana de Saúde 2004. [acesso 20 ago. 2008]. Disponível em: http://www.opas.org.br/medicamentos/temas_documentos_detalhe.cfm?id $=46 \&$ iddoc $=274$

20. Monteiro MF, Filho DCS. Exercício físico e controle da pressão arterial. Rev Bras Med Esporte. 2004;10(6):513-16.

21. Ramos JGL, Costa SM, Francisco MA, Almeida AG, Machado MF, Borowski C. É seguro não utilizar sulfato de magnésio nas pacientes com pré-eclampsia? Rev Bras Ginecol Obstet. 2000;22(1):13-7.

22. Pafenbarger RS Jr. Contributions of epidemiology to exercise science and cardiovascular health. Med Sci Sports Exerc. 1988;20(5):426-38.

23. American College of Sports Medicine. Exercise and physical activity for older adults. Med Sci Sports Exerc 1998;30(6):992-1008.

24. Kraemer WJ, Adams K, Cafarelli E, Dudley GA, Dooly C, Feigenbaum MS. American College of Sports Medicine. Progression models in resistance training for healthy adults. Med Sci Sports Exerc. 2002;34(2):364-80.

25. Pollock M, Franklin B, Balady G, Chaitman B, Fleg J, Fletcher B, et al. Resistance exercise in individuals with and without cardiovascular disease. Circulation. 2000;101(7):828-33.

26. Simão R, Polito MD, Lemos A. Duplo-produto em exercícios contra-resistidos. Fit Perfor J. 2003;2(5):279-84.

27. Farinatti PTV, Assis BFCB. Estudo da freqüência cardíaca, pressão arterial e duplo- produto em exercícios contraresistência e aeróbico contínuo. Rev Bras Ativ Física Saúde. 2000;5:5-16.

28. Ganem EM, Castiglia YMM. Anestesia na pré-eclampsia. Rev Bras Anestesiol. 2002;52(4):481-97. 
29. Pivarnik JM, Ayres NA, Mauer MB. Effects of maternal aerobic fitness on cardiorespiratory responses to exercise. Med Sci Sports Exerc. 2003;25(9):993-8.

30. American College of Obstetricians and Gynecologists. Committee Opinion n. 267. Exercise during pregnancy and the postpartum period. Clin Obstet Gynecol. 2003;46(2):496-9.

31. Sheldahl LM, Wann LS, Clifford PS, Tristani FE, Wolf LG, Kalbfleisch JH. Effect of central hypervolemia on cardiac performance during exercise. J Appl Physiol. 20044;57(6):1662-7.

32. Epstein M. Renal effects of head-out waterimmersion in humans: a 15-year update. Physiol Rev. 2002;72(3):563-621.

33. Hartman S, Kolble N, Rake A, Bung P, Huch A, Ruch R. Aqua fit during pregnancy: maternal and fetal hemodynamic responses during rest, immersion and exercise. Geburtshilfe und Frauenheilkunde. 2001;61(12):977-82.

34. McMurray RG, Katz VL, Berry MJ, Cefalo RC. Cardiovascular responses of pregnant women during aerobic exercise in water: a longitudinal study. Int J Sports Med. 1998;9(6):443-7.

35. Moutquin JM, Peter G, Burrows RF, Rey E, Helewa ME, Lange IR. Report of the Canadian Hypertension Society Consensus Conference: 2. Nonpharmacologic management and prevention of hypertensive disorders in pregnancy. Can Med Assoc J. 1998;157(7):907-19.

36. Tomoda S, Ogita S, Tamura T. Exercise therapy for the prevention of gestational hypertension. J Soc Gynecol Investig. 1998;3(2):304.

37. Sorensen TK, Williams MA, Lee IM, Dashow EE, Thompson ML, Luthy DA. Recreational physical activity during pregnancy and risk of preeclampsia. Hypertension. 2003;41(6):1273-80.

38. Weissgerber TL, Wolfe LA, Davies GAL. The role of regular physical activity in pre-eclampsia prevention. Med Sci Sports Exerc. 2004;36(12):2024-31.

39. Jürimäe T, Jürimäe J, Pihl E. Circulatory response to single circuit weight and walking training session of similar energy cost in middle-age overweight females. Clin Physiol. 2000;20(2):143-9.

40. Farinatti PTV, Assis BFCB. Estudo da freqüência cardíaca, pressão arterial e duplo-produto em exercícios contraresistência e aeróbico contínuo. Rev Bras Ativ Física Saúde. 2000;5:5-16.

Recebido: 29/09/2008

Received: 09/29/2008

Aprovado: $27 / 07 / 2010$

Approved: 07/27/2010 